\title{
Effect of excitation on gas centered swirl coaxial injectors
}

\author{
Gujeong Park *1, Sukil Oh${ }^{1}$, Jinhyun Bae ${ }^{1}$, Youngbin Yoon ${ }^{1}$ \\ ${ }^{1}$ Department of Mechanical and Aerospace Engineering, Seoul National University, Republic \\ of Korea \\ *Corresponding author: ybyoon@snu.ac.kr
}

\begin{abstract}
Studies on combustion instability in liquid rocket engines are important in improving combustion efficiency and preventing combustion chamber losses. To prevent combustion instability, methods such as baffles and cavities are used. The injector is located in the middle of the perturbation-propagation process in the rocket engine, so it is important to study the suppression of combustion instability using the design of the injector. Much research has been focused on the study of liquid excitation in a single injector; however, the actual injector used in a liquid rocket engine is a coaxial injector. In this study, the dynamic characteristics of a gas-centred swirl coaxial injector were investigated by varying the gap thickness and momentum-flux ratio. Spray photographs were captured by synchronizing a stroboscope and digital camera, and a high-speed camera and Xenon lamp were also used. To measure the liquid film, a measurement system was implemented using the electrical conductance method. For excitation of the gas, an acoustic speaker was used to impart a frequency to the gas. The gGas velocity and effect of excitation were measured by hot-wire anemometry. A mechanical pulsator was used for liquid flow excitation. Liquid fluctuation was measured by a dynamic pressure sensor. In both gas and liquid excitation cases, the gain increased as the gap thickness decreased and the momentum-flux ratio increased. From these results, it can be concluded that gap thickness and momentum-flux ratio are major factors in suppressing combustion instability.
\end{abstract}

\section{Keywords}

Gas-centred Swirl Coaxial Injector, Excitation, Gain

\section{Introduction}

Research on combustion efficiency and combustion instability is important in the development of a liquid rocket engine. Combustion instability occurs in the combustion chamber when the combustion and the flow of the propellant supply system are combined. This not only reduces combustion efficiency, but also leads to combustion chamber losses in extreme cases. Disturbances that can occur in propellant supply systems ranges from a few $\mathrm{Hz}$ to a few thousand $\mathrm{Hz}$, depending on the causes [1].

One of the causes of combustion instability is perturbations in the flow rate of the propellant to the combustion chamber, which can be caused by various factors. This disturbance of the flow travels through the feed line, the injector, and the combustion chamber, affecting the stability of the combustion. The pressure perturbation resulting from combustion instability can also affect the propellant flow in the feed system. Therefore, the design of an injector located in the middle of this process is of considerable importance. If the injector can serve as a shock absorber to reduce the disturbance from the supply line by optimized design, it would be able to prevent instability of the spray, which could cause combustion instability.

Research into the suppression of combustion instability through injector geometry has been going on since the 1990s. In 1996, Bazarov et al. found that the injector dynamics affected the instability of the liquid rocket engine [2]. In 2007, Soller et al. investigated the combustion instability of an oxidant-feed system and combustion chamber in various gas centred swirl coaxial injectors. They confirmed that the perturbation of the oxidant supply system affects the perturbation of the combustion chamber [3]. Heister et al. proposed several models of selfresonance phenomena of a close-type single swirl injector and analysed it numerically [4]. Fu et al. confirmed the dynamic characteristics of an open-type single swirl injector by changing the injector geometry using a mechanical pulsator.

The previous research only focused on the response characteristics to liquid disturbance in a single swirl injector. The phenomenon of propellant disturbance in a gas-liquid injector used in a liquid rocket engine has not been extensively studied. In this study, the dynamic characteristics of the spray from disturbances of the gas and the liquid were investigated by varying the gap thickness of the gas centred swirl coaxial injector, used mainly in the Russian multi-stage combustion cycle liquid rocket engine, such as the RD-170. 


\section{Material and methods}

The experimental setup is shown in Fig. 1. In this experiment, air and water were used as the experimental fluids to represent oxidant and fuel, respectively. The apparatus comprises a function generator, a data acquisition system (DAQ), an injector, and a high-speed camera. The function generator supplied a constant frequency, and the voltage for the liquid film thickness was measured by the electrodes in the orifice. The method of measuring the liquid film thickness with electrodes, using the electrical conductivity of water, was proposed by Suyari and Lefebvre [5]. Two thin electrodes were placed at the end of the orifice to obtain the voltage generated by the liquid film thickness. The manifold pressure of the injector was measured by a static- and a dynamic-pressure sensor, and was recorded by the DAQ system. The spray image of the injector was obtained using a high-speed camera and a DSLR camera.

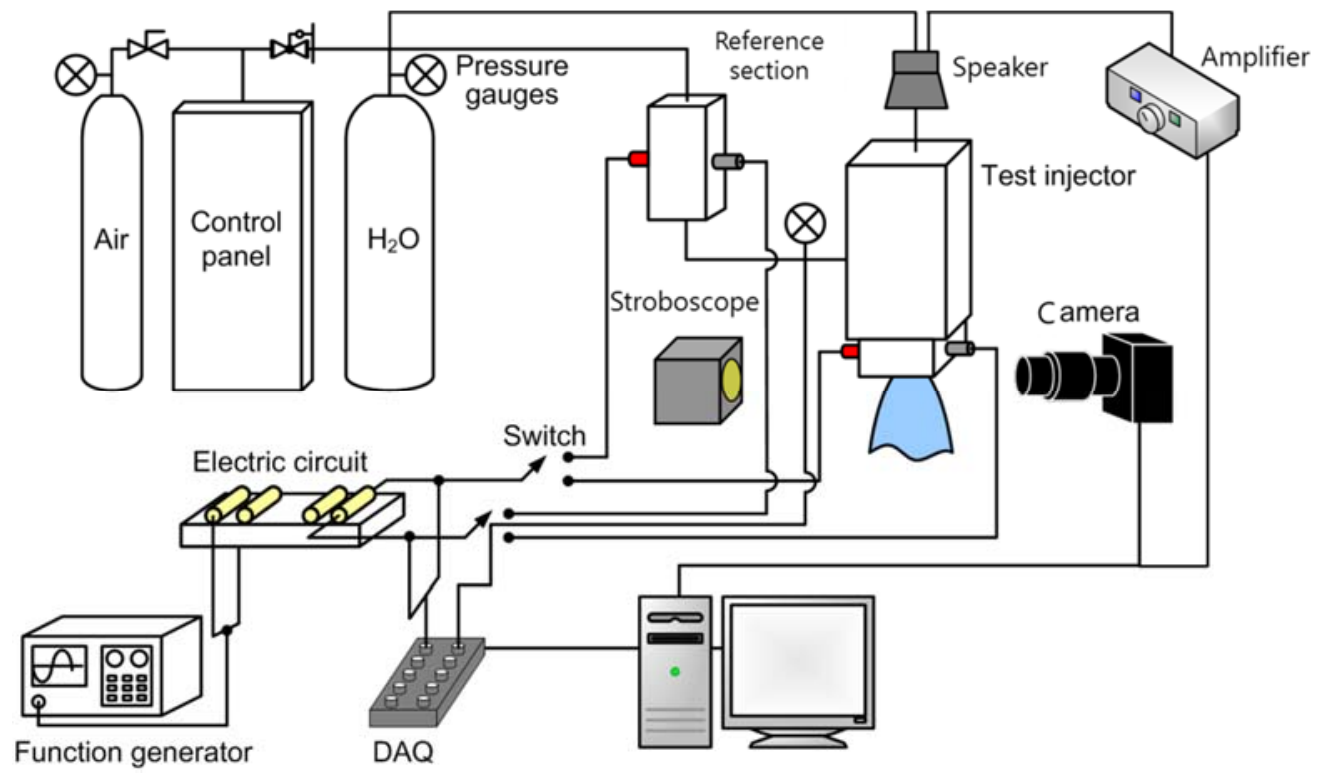

Figure 1. Experimental apparatus

For the gas excitation, a speaker, amplified by an amplifier, was installed in the middle of the gas supply line to generate the perturbation of the gas flow. The influence of the gas velocity perturbation was measured by using hot-wire anemometry. For the liquid excitation, a mechanical pulsator was installed in the middle of the liquid supply line to generate perturbations in the pressure of the supplied liquid. The pulsator was designed to change the area of the flow path by rotating a plate at a predetermined speed, up to $1000 \mathrm{~Hz}$.

The geometry of the gas-centred swirl injector used in the experiment is shown in Fig. 2. The gas orifice is located in the middle of the injector, surrounded by the liquid orifice. The gas is jetted and the liquid is swirled through the tangential inlet and injected. The diameter of the injector orifice was $8 \mathrm{~mm}\left(2 R_{n}\right)$ and the gas orifice diameter $\left(D_{g}\right)$ was $6 \mathrm{~mm}$. The tangential inlet diameter ( $\left.R_{\text {inlet }}\right)$ was $0.9 \mathrm{~mm}$ for the gas flow perturbation case, and $1.5 \mathrm{~mm}$ for the liquid flow perturbation case. The gap thickness ( $h_{\text {gap }}$ ) was set at $0.3 \mathrm{~mm}, 0.5 \mathrm{~mm}$, and $0.7 \mathrm{~mm}$.

In this experiment, the speaker frequency was varied from $200-1100 \mathrm{~Hz}$ at $100 \mathrm{~Hz}$ intervals to simulate disturbances in the oxidant excess gas. The liquid fuel disturbances were simulated by varying the pulsator frequency from $200-1000 \mathrm{~Hz}$ in $50 \mathrm{~Hz}$ increments. Experiments were carried out by setting the gap thickness, which influences the gas-liquid mixing and the gas-liquid momentum-flux ratio as the main parameter in the gasliquid injector. The momentum flux ratio is defined by Eq. 1, and the experimental conditions are shown in Table 1.

$$
M R=\frac{\rho_{g} U_{g}^{2}}{\rho_{l} U_{a l}^{2}}
$$




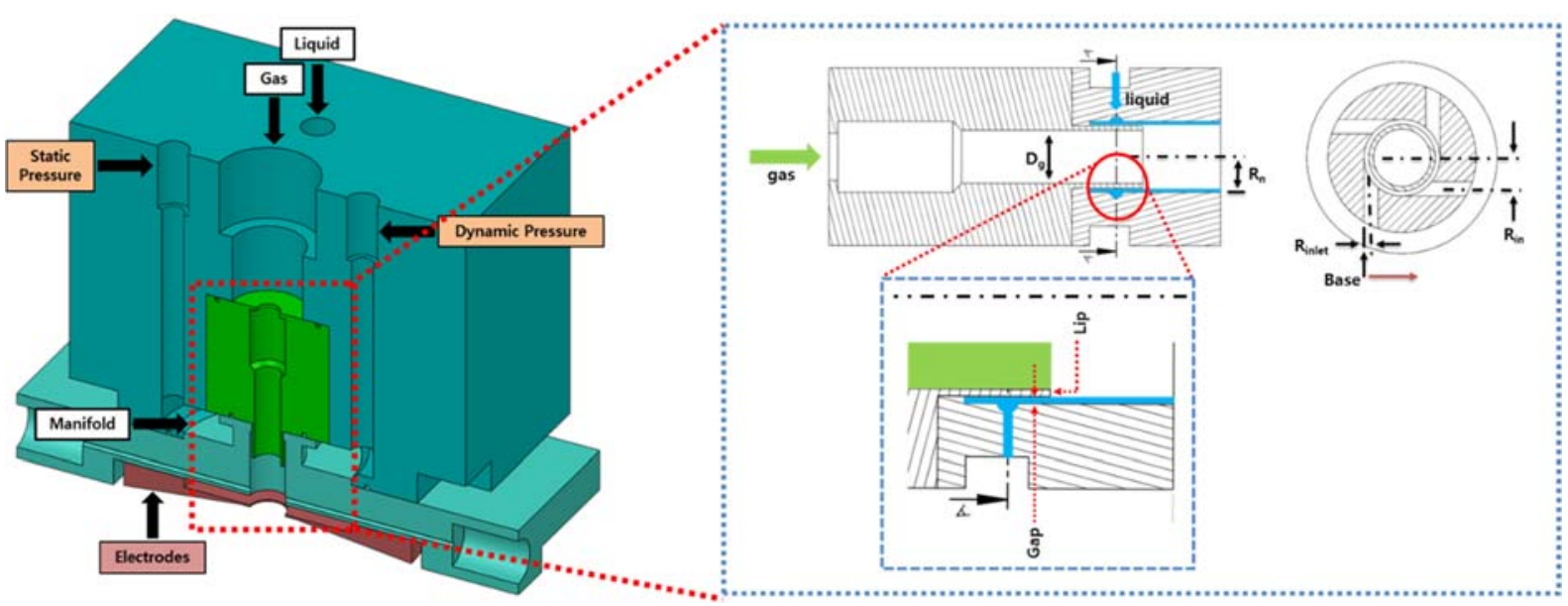

Figure 2. Geometry of gas centred swirl coaxial injector

Table 1. Experimental condition.

\begin{tabular}{c|cc}
\hline \hline & Gas Excitation & Liquid Excitation \\
\hline \hline Gap thickness $[\mathrm{mm}]$ & \multicolumn{2}{|c}{$0.3,0.5,0.7$} \\
Momentum flux ratio & \multicolumn{2}{|c}{$0,0.5,1,2$} \\
Input Frequency $[\mathrm{Hz}]$ & $200-1100$ & $200-1000$ \\
Tangential inlet diameter $[\mathrm{mm}]$ & 0.9 & 1.5
\end{tabular}

\section{Results and discussion}

The thickness of the liquid film at the end of the injector orifice is an important factor influencing the spray angle, breakup length, and droplet size [6]. Therefore, understanding the change in liquid film thickness at the end of the orifice in response to the generated flow disturbances is important in terms of spray- and combustion-instability predictions. In this experiment, the response characteristics of the liquid film thickness to the perturbation during gas excitation and liquid excitation is expressed by the injector transfer function as shown in Eqs 2-4.

$I T F_{\text {gas }}=\frac{\text { output }}{\text { Input }}=\frac{t^{\prime} / \bar{T}}{u^{\prime} / \bar{U}}$

$I T F_{\text {liquid }}=\frac{\text { Output }}{\text { Input }}=\frac{t^{\prime} / \bar{T}}{p^{\prime} / \bar{P}}$

gain $=|\mathrm{ITF}|$

In a gas centred swirl coaxial injector, the gap thickness is a geometric factor that determines the liquid film thickness in a liquid flow. Fig. 3 shows the results of the injector dynamic characteristics for varying gap thickness when the gas is excited while the gas and liquid are sprayed at the same time. As the gap thickness decreased, the gain increased. A decrease in the gap thickness means an increase in the lip thickness because the diameter of the gas and liquid orifices is kept constant. Larger lip thickness produces greater flow eddy [7]. Therefore, when the gap is thinner, i.e., when the lip is thicker, a large vortex forms, and the magnitude of the perturbation becomes larger. 


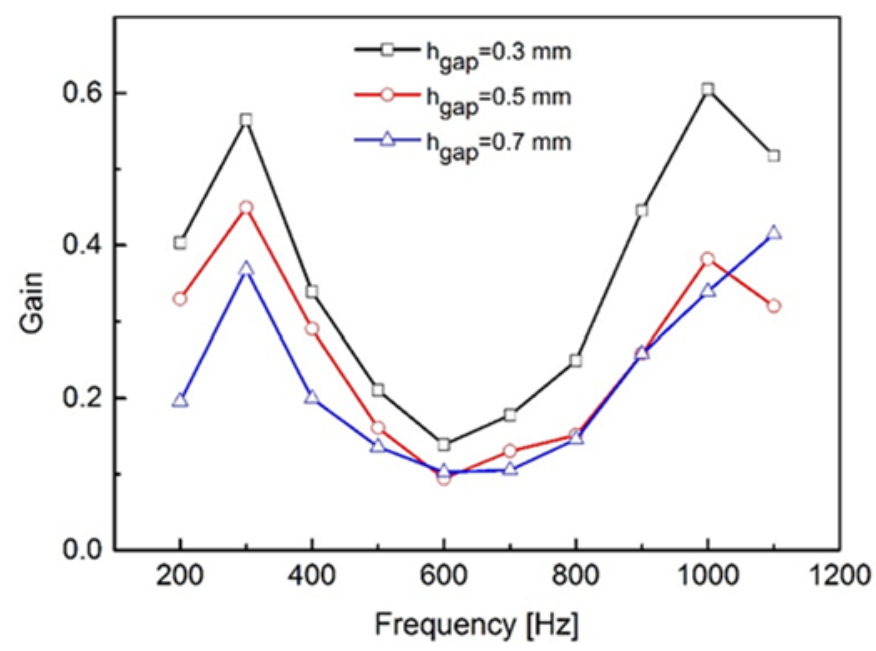

Figure 3. Dynamic characteristics for varying gap thickness with gas flow perturbations

Figure 4 shows the response characteristics of the spray when only the gas is excited, the liquid flow rate is fixed, and the gas-liquid momentum-flux ratio is changed. The increase in the gas-liquid momentum-flux ratio means that the momentum of the gas relative to that of the liquid increases, resulting in a larger momentum transfer for the same mixing-zone length. Therefore, more gas velocity perturbations are transmitted to the liquid, which increases the liquid film tremor at the end of the orifice. However, it can be seen that the increase of the gain due to the increase of the momentum-flux ratio changes with frequency. The effect of the spray pattern on frequency is shown in Fig. 5. When the magnitude of the gain is larger, the liquid film thickness reduces because the effect of the excitation acted on strongly liquid flow. Thus, it can be seen that the spray breaks up quicker and has a qualitatively wider angle due to its earlier breakup.

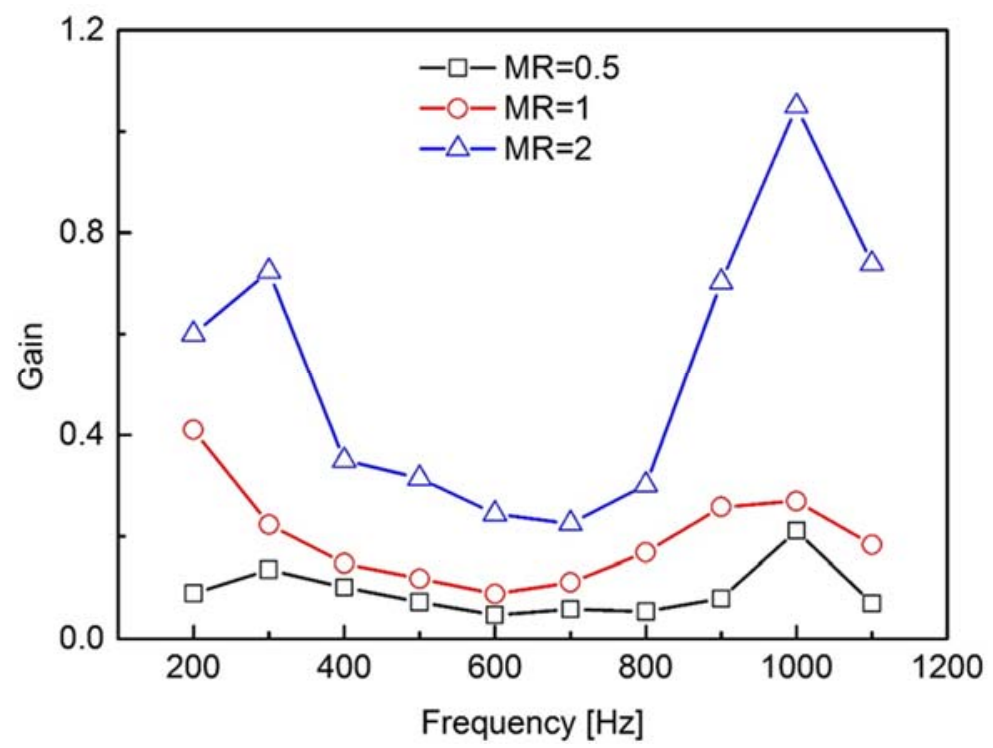

Figure 4. Dynamic characteristics for varying momentum-flux ratios with gas flow perturbations 


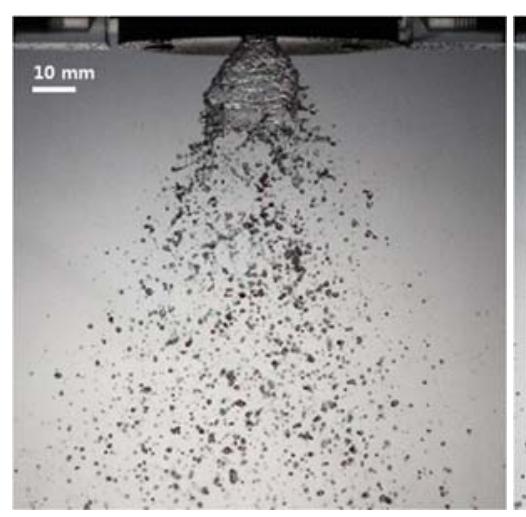

(a)

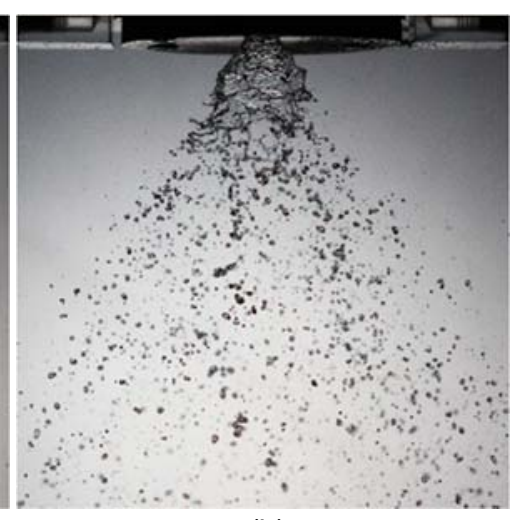

(b)

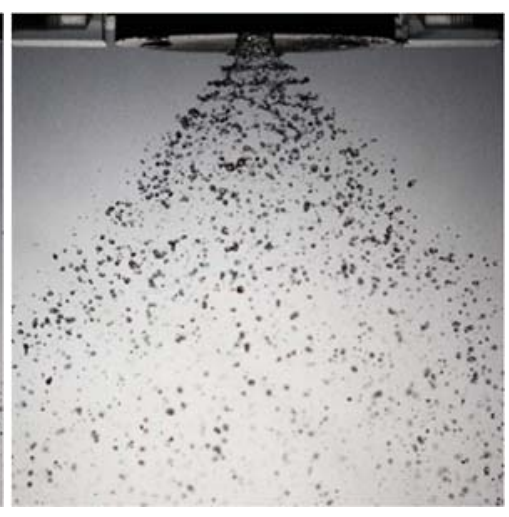

(c)

Figure 5. Spray pattern with gas excitation for $h_{\text {gap }}=0.5 \mathrm{~mm}$ and $M R=2 ;$ (a) $f=0 \mathrm{~Hz}$, (b) $f=600 \mathrm{~Hz}$, and (c) $f=1000 \mathrm{~Hz}$

\section{Liquid Excitation}

Figure 6 shows the dynamic characteristics of the injector for the three gap thicknesses with liquid excitation, while the liquid flow rate is fixed without injecting gas. Gain changes and a phenomenon in which the gain decreased at a specific frequency (sharp drop) can be observed. When the gap thickness was $0.3 \mathrm{~mm}$, the gain was more than twice that of the other cases, which is thought to be due to the liquid film thickness. As shown in Table 2, when the gap thickness was $0.3 \mathrm{~mm}$, the liquid film thickness was approximately half that of the other two gaps. Such a thin liquid film is susceptible to tremors, so that the change in thickness was doubled due to the influence of the excitation. As the gap thickness increased, the frequency at which the gain decreased also decreased. This is because the gap thickness acts as a low pass filter; the fluid passing through the tangential inlet meets the gap, which is a suddenly enlarged space, and only the low-frequency fluid passes through.

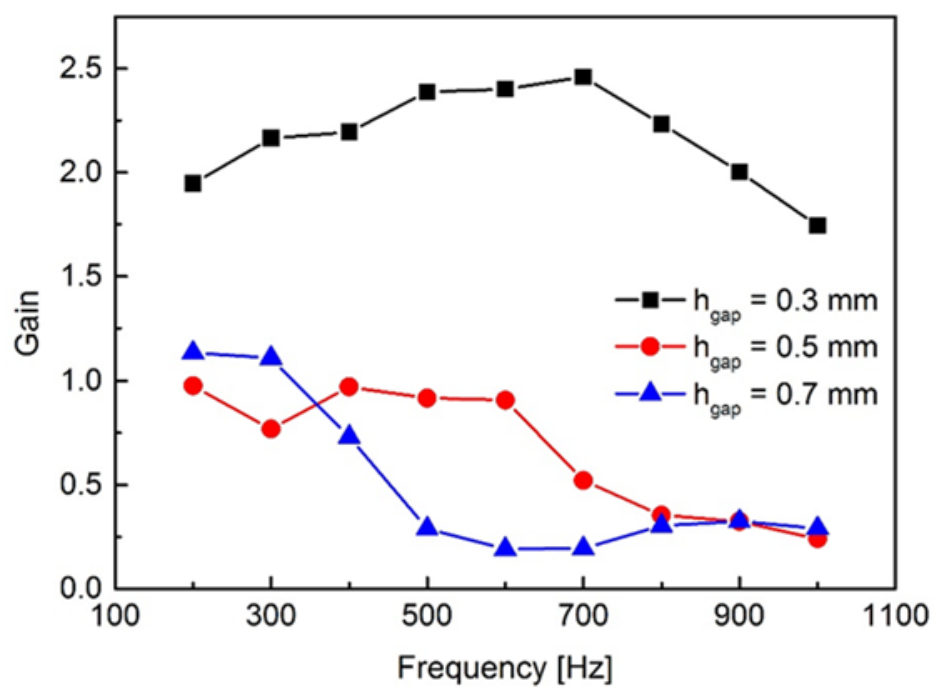

Figure 6. Dynamic characteristics for varying gap thickness with liquid flow perturbations

Table 2. Film thickness and film thickness fluctuation for varying gap thicknesses.

\begin{tabular}{c|c|c}
\hline \hline $\begin{array}{c}\text { Gap thickness } \\
{[\mathrm{mm}]}\end{array}$ & $\begin{array}{c}\text { Film thickness } \\
@ 300 \mathrm{~Hz}[\mu \mathrm{m}]\end{array}$ & $\begin{array}{c}\text { Film thickness fluctuation } \\
@ 300 \mathrm{~Hz}[\mu \mathrm{m}]\end{array}$ \\
\hline 0.3 & 480.70 & 55.84 \\
0.5 & 714.82 & 26.97 \\
0.7 & 887.77 & 29.21
\end{tabular}

Figure 7 shows the results of the dynamic characteristics with different gas-liquid momentum-flux ratios when the gas and the liquid were injected simultaneously, with only the liquid having flow perturbations. An increase in the 
gas-liquid momentum-flux ratio leads to the formation of a thin liquid film thickness that is susceptible to perturbations, thereby increasing the gain. It was also considered that the gas momentum added to the liquid tremor, and increased the gain. Figure 8 shows the spray pattern at three different frequencies. It can be seen that the spray has a clear single layer of the liquid mass concentrated at every excitation period in the case where the gain was larger, similarly to the case of gas flow perturbations.

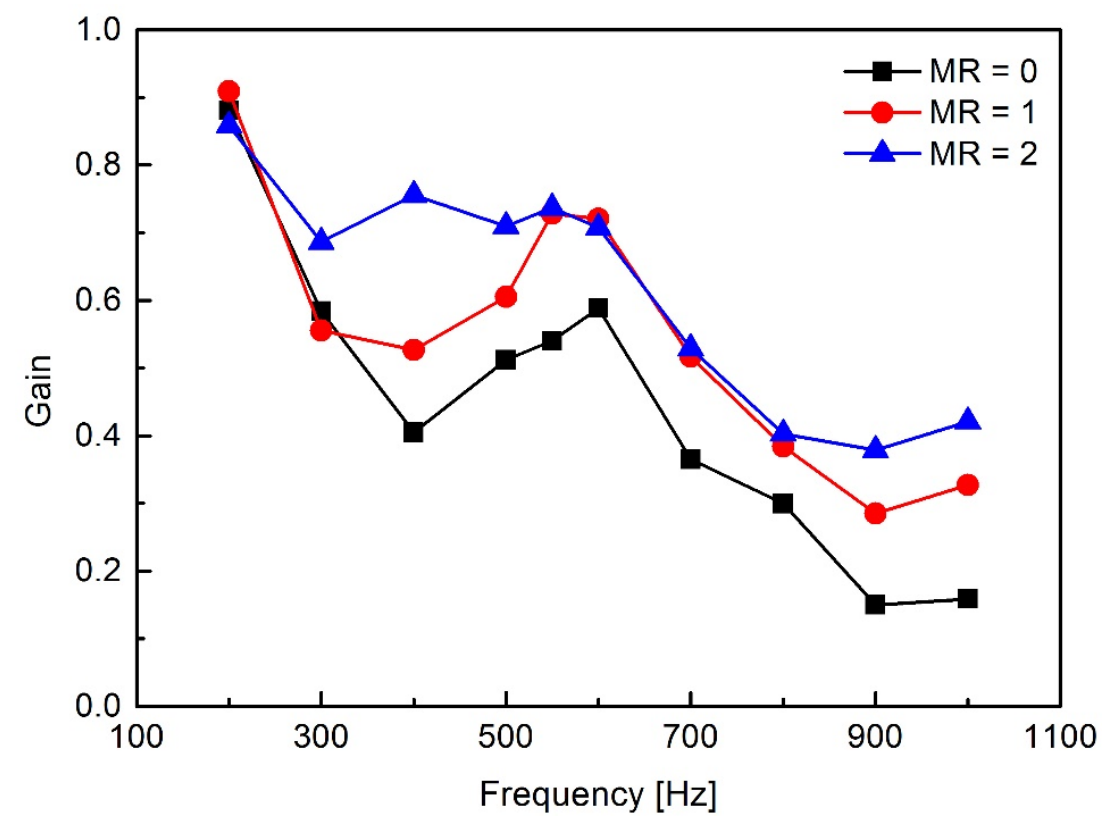

Figure 7. Dynamic characteristics for varying momentum-flux ratios with liquid flow perturbations

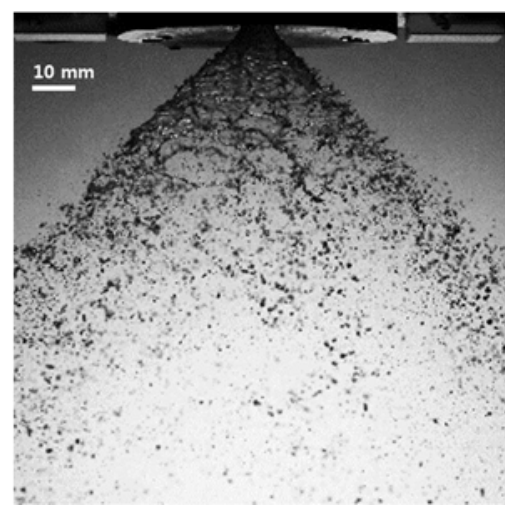

(a)

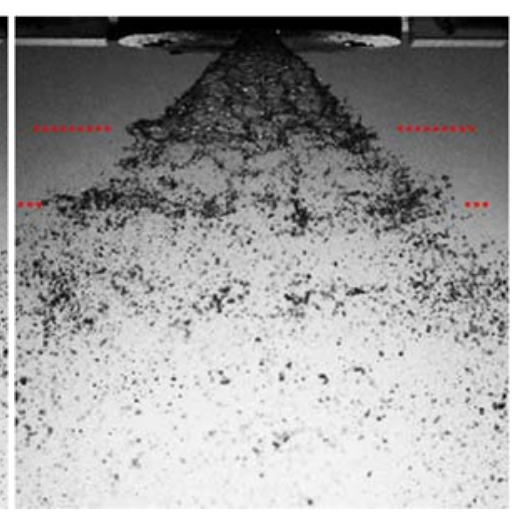

(b)

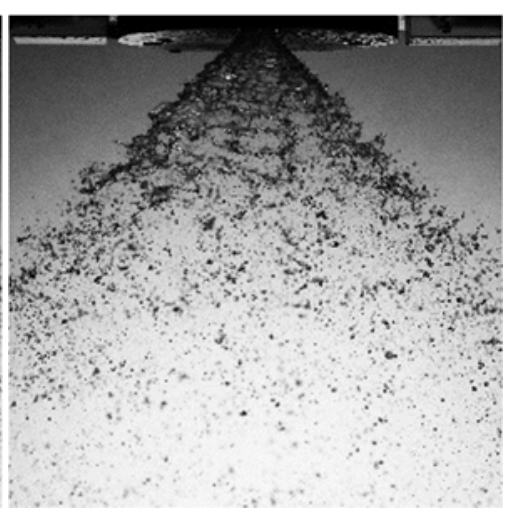

(c)

Figure 8. Spray pattern with liquid excitation for $h_{\text {gap }}=0.5 \mathrm{~mm}$ and $M R=2 ;(a) f=0 \mathrm{~Hz}$, (b) $f=400 \mathrm{~Hz}$, and (c) $f=900 \mathrm{~Hz}$

\section{Conclusions}

To investigate the dynamic characteristics of a gas-centred swirl coaxial injector, which is used mainly in a multistage combustion cycle engine, a speaker and a mechanical pulsator were used to generate gas- and liquid-flow perturbations. The effect of the geometry on the dynamic characteristics was investigated by varying the gap thickness, and the dynamic characteristics of the injector were determined by varying the momentum flux ratio. In the case of gas excitation, the gain increased as the gap thickness decreased. It is considered that as the gap thickness decreased and the lip became thicker, a larger vortex formed, resulting in a larger gain. As the momentum flux ratio increased, the gain increased. This is because more of the gas momentum was transferred to the liquid, as the momentum of the gas relative to that of the liquid increased.

In the case of liquid excitation, the gain increased as the gap thickness decreased. Because of the spatial limitations of the smaller gap thickness, a thin liquid film was formed and was susceptible to tremors, which are caused by the influence of the excitation. As the momentum flux ratio increased, the gain increased. This is thought to be due to the momentum of the gas being transmitted to the liquid. 
From these results, it can be concluded that the gap thickness is considered to be the main geometric factor for suppressing combustion instability, and that the momentum-flux ratio was also a factor for suppressing combustion instability.

\section{Acknowledgements}

This work was supported by the Advanced Research Centre Program (NRF-2013R1A5A1073861) through a National Research Foundation of Korea (NRF) grant, funded by the Korean government (MSIP), and contracted through the Advanced Space Propulsion Research Centre at Seoul National University and was supported by an NRF (National Research Foundation of Korea) Grant funded by the Korean Government (NRF-2016-Fostering Core Leaders of the Future Basic Science Program/Global Ph.D. Fellowship Program).

\section{Nomenclature}

ITF injector transfer function

t' film thickness fluctuation $[\mu \mathrm{m}]$

$\bar{T} \quad$ average of film thickness $[\mu \mathrm{m}]$

p' manifold pressure fluctuation [bar]

$\bar{P} \quad$ average of manifold fluctuation [bar]

MR momentum flux ratio

$\rho_{g} \quad$ density of gas

$\rho_{l} \quad$ density of liquid

u' gas velocity fluctuation [m/s]

$\bar{U} \quad$ average of gas velocity [m/s]

$U_{g} \quad$ velocity of gas

$U_{a l} \quad$ axial velocity of liquid

$\mathrm{D}_{\mathrm{g}} \quad$ diameter of the gas orifice

$R_{\text {in }} \quad$ distance from the centre of the injector orifice to the centre of the tangential inlet

$\mathrm{R}_{\mathrm{n}} \quad$ radius of the injector orifice

$\mathrm{R}_{\text {inlet }} \quad$ radius of the tangential inlet

\section{References}

[1] Harrje, D. T., and Reardon, F. H., 1972, NASA Rept. SP-194.

[2] Bazarov, V. G., and Yang, V., 1998, Journal of Propulsion and Power, 14 (5).

[3] Soller, S., Wagner, R., Kau, H., Martin, P., and Mading, C., 2007, 43rd AIAA/ASME/SAE/ASEE Joint Propulsion

Conference and Exhibit.

[4] Ahn, B., Ismaliov, M., and Heister, S. D., 2009, 45th AIAA/ASME/ASE/ASEE Joint Propulsion Conference \& Exhibit.

[5] Suyari, M., and Lefebvre, A. H., 1986, Journal of Propulsion and Power, 2 (6), pp. 528-533

[6] Bayvel, L., and Orzechowski, Z., 1993, Liquid Atomization.

[7] Tsohas, J., Canino, J. V., and Heister, S. D., 2007, 43rd AIAA/ASME/SAE/ASEE Joint Propulsion Conference and Exhibit. 